\title{
Summaries of the FY 1981 Applied Mathematical Sciences \\ Research Program
}

December 1981

DOE/ER--0115

DE82 007364

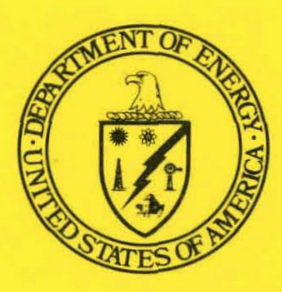

U.S. Department of Energy

Office of Energy Research

Office of Basic Energy Sciences

Division of Engineering, Mathematical

and Geosciences 


\section{DISCLAIMER}

This report was prepared as an account of work sponsored by an agency of the United States Government. Neither the United States Government nor any agency Thereof, nor any of their employees, makes any warranty, express or implied, or assumes any legal liability or responsibility for the accuracy, completeness, or usefulness of any information, apparatus, product, or process disclosed, or represents that its use would not infringe privately owned rights. Reference herein to any specific commercial product, process, or service by trade name, trademark, manufacturer, or otherwise does not necessarily constitute or imply its endorsement, recommendation, or favoring by the United States Government or any agency thereof. The views and opinions of authors expressed herein do not necessarily state or reflect those of the United States Government or any agency thereof. 


\section{DISCLAIMER}

Portions of this document may be illegible in electronic image products. Images are produced from the best available original document. 


\title{
DISCLAIMER
}

\begin{abstract}
-'Ihis report was prepared as an accuunt of work sponsored by an agency of the United States Government. Neither the United States Government nor any agency thereof, nor any of their employees, makes any warranty, express or implied, or assumes any legal liability or responsibility for the accuracy, completeness, or usefulness of any information, apparatus, product, or process disclosed, or represents that its use would not infringe privately owned rights. Reference herein to any specific commercial product, process, or service by trade name, trademark, manufacturer, or otherwise, docs not necessarily constitute or imply its endorsement, recommendation, or favoring by the United States Government or any agency thereof. The views and opinions of authors expressed herein do not necessarily state or reflect those of the United States Government or any agency thereof."
\end{abstract}

This report has been reproduced directly from the best available copy.

Available from the National Technical Information Servire, I. S. Department of Commerce, Springfield, Virginia 22161.

Price: Printed Copy A04

Microfiche A01

Codes are used for pricing all publications. The code is determined by the number of pages in the publication. Information pertaining to the pricing codes can be found in the current issues of the following publications, which are generally available in most libraries: Energy Research Abstracts, (ERA); Government Reports Announcements and Index (GRA and I); Scientific and Technical Abstract Reports (STAR); and publication, NTIS-PR-360 available from (NTIS) at the above address. 


\section{Summaries of the FY 1981 Applied Mathematical Sciences Research Program}

December 1981
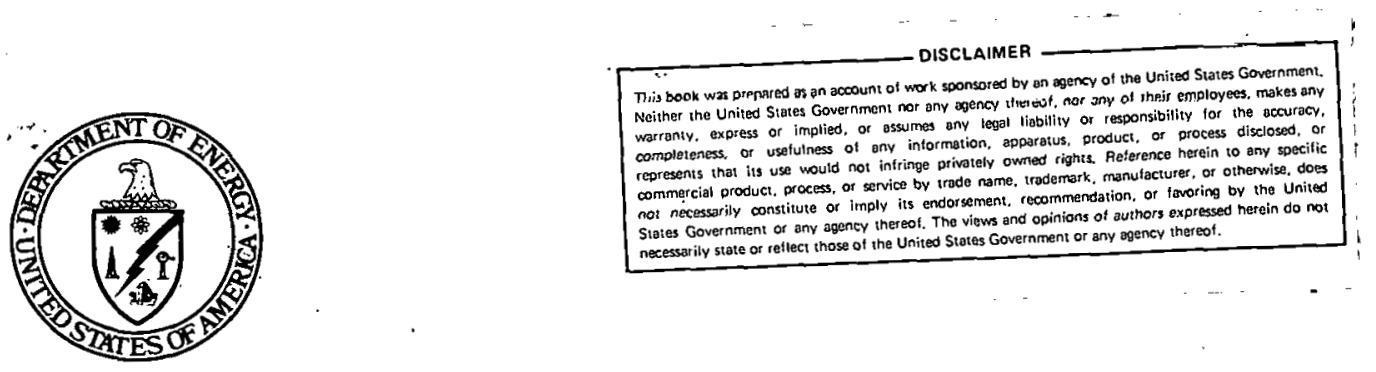

\section{U.S. Department of Energy}

Office of Energy Research Office of Basic Energy Sciences

Division of Engineering, Mathematical and Geosciences

Washington, D.C. 20545 


\section{Table of Contents}

Introduction 2

Program Overview 3

Ames Laboratory 9

Argonne National Laboratory 10

Brookhaven National Laboratory 12

California Institute of Technology 14

George Washington University 15

Kent State University 16

La Jolla Institute $\quad 17$

Los Alamos National Laboratory 18

Lawrence Berkeley Laboratory 20

Lawrence Livermore National Laboratory 22

Massachusetts Institute of Technology 24

Northwestern University 25

New York University- Courant Institute 26

Oak Ridge National Laboratory 35

Paclfic Northwest Laboratory - Battelle 37

Princeton University $\quad 39$

Purdue University $\quad 40$

Rice University 41

Science Applications. Inc 42

Sandia National Laboratories. Albuquerque 43

Sandia National Laboratories. Livermore 44

Stanford University $\quad 45$

Stanford University $\quad 46$

Stanford University $\quad 47$

Texas Tech University 48

University of California. Berkeley 49

University of California. Los Angeles 50

University of Colorado 51

Universlty of Delaware 52

University of Florida 53

University of llinols 54

University of Illinois $\quad 55$

University of Texas $\quad 56$

University of Texas 57

University of Wisconsin . 58

Virginia Polytechnic Institute 59

Yale University . 60 


\section{INTRODUCTION}

The purpose of this report is to provide a convenient compllation of summaries of the individual research projects that constitute the Applied Mathematical Sclences program in the Office of Basic Energy Sciences. Office of Energy Research. Department of Energy.

The report is ordered alphabetically by institution. DOE laboratories are integrated in alphabetical order with university and other non-DOE institutional participants in the program.

In sequence, each project entry begins with an underlined identification number. followed by the project title. the FY 1981 funds allocated. and the program component identification number:

01 Analytic and Numerical Methods Includes. Applied Analysis, Computational Mathematics, and Numerical Methods for the solution of Partial Differential Equations

02 Information Analysis Techniques

Includes Statistical Methods. Data Management Technlques, and Display and Analysis Techniques

03 Aovániced Computing Concepts

Includes Software Engineering. Distributed Systems. and High Performance Systems

04 Special Projects

Includes operating and maintenance of Research Computing Facillties and workshops and studies. 


\author{
U. S. Department of Energy \\ Office of Energy Research \\ Office of Basic Energy Sciences \\ Division of Engineering. Mathematical and Geosciences \\ APPLIED MATHEMATICAL SCIENCES
}

\begin{abstract}
Program Overview
Applied Mathematical Sciences serves as the DOE focal point for monitoring and advancing the state of the art in mathematics. statistics. and computer science. Several DOE mission programs develop and refine specific techniques from the applied mathematical sciences applicable to their immediate needs. In contrast. Applied Mathematical Sciences concentrates on more broadly based. continuing needs throughout the DOE community. Emphasis is placed on research basic to the analysis. development, and use of large-scale computational models: the management and analysis of large. complex collections of information: and the effective use of DOE computing resources. The purpose of this research is not to improve existing technologies and methodologies. but rather to render them obsolete.
\end{abstract}

Each part of the Applied Mathematical Sciences activity has been designed with the help and advice of leading mathematicians and computer scientists from universities, industry, and DOE laboratories to assure the broadest and greatest impact on the nation's energy R\&D enterprise. Many of them are expert in industry's needs in the relevant areas. Close llaison is maintained with other Federal agencles in the selection of areas of emphasis and of individual research tasks. This is high leverage research. In favorable cases. the results may be of great benefit simultaneously to a number of different energy technologies. The requested increase will be an exceptionally sound investment.

The FY 1981 budget for Applied Mathematical Sciences is $\$ 11,370,000$, a $\$ 1.120 .000$ increase over the amount appropriated in FY 1980. Corresponding to the above areas of emphasis, the Applied Mathematical Sciences research program has been organized into four subactivities: Analytical and Numerical Methods, Information Analysis Techniques, Advanced Computer Concepts, and Special Projects.

\title{
Analytical and Numerical Methods
}

In FY 1981. \$5,900,000 was allocated for Analytical and Numerical Methods. This research addresses questions basic to improved predictions of the feasibility. reliability. safety. and efficiency of energy. systems. Many results are also applicable to the simulation of environmental or health effects and to modeling energy supply-demand. Analytical and Numerical Methods comprises three categories of research activities reflecting the principal thrust of the program in previous years: Applied Analysis, Computational Mathematics, and Numerical Methods for Partial Differential Equations.

Applied Analysis concentrates on techniques to develop and analyze mathematical models of scientific. engineering. and socio-economic phenomena. It aiso includes the analysis of the transition from a mathematical model to corresponding approximate computational models. The nature of the research in Applied Analysis is well illustrated by the following significant achlevements.

Recently. In the search for combustors in which fuels are burned cleanly and efficiently. mathematiclans as Northwestern University set up a 
theoretical model for a particular type of gas burner. Their studies have led to predictions of flame speeds and temperatures as a function of gas inflow. These predictions were later confirmed by independent experiments at Sandia National Laboratory's combustion diagnostics facility. This collaborative effort is expected to have a significant impact on the design of environmentally sound combustors for efficient utilization of fossil fuels.

In a quite different area, a major breakthrough in the analysis of binary alloy solidification has been accomplished at ORNL. The new successful approach to this problem is due to a novel application of the first law of thermodynamics (energy conservation law) to the solution of a classical problem in mathematics - viz. diffusion across a moving boundary. This advance will make possible the effective computer simulation of binary alloy solidification processes for industrial and energy related metalturgical applications.

Computational Mathematics involves the design and analysis of algorithms for basic computational problems (A.g., linear algebra, optimization. approximation and interpolation, and differential equations). Emphiasls is also placed on the implementation of the resulting algorithms in transportable. high-quality sultware sultable for software libraries.

The following example demonstrates accomplishments in the understanding of linear programming techniques vital to econometric modellng. Much publicity was generated in the autumn of 1979 concerning an algorithm developed by the Russian mathematician Khachiyan that was able to solve the linear programming problem in polynomial time. The impression was given that this algorithm (which has come to be called the "Russian" or "ellipsoid" algorithm) would revolutionize solution techniques for many problems that had previously been considered intractable. In the ensuing year. many researchers have studied ellipsoid algorithms. including mathematicians and computer scientists at the Systems Optimization Laboratory. Stanford University. It has been shown that the polynomial bound on the number of iterations of an ellipsoid method is extremely large. even for problems of moderate size. This is impnriant hecause in practice tho pcrformance of ellipsoid inthiuus invariably approaches the worst case. (By contrast. the number of lterations required in practice by the simplex method is a linear function of the problem size, despite an exponential bound in the worst case.) A detailed numerical investigation of ellipsoid atgorithms and numerous variants has revealed serlous practical difficulties. since the ellipsold cannot be represented compactly for large-scale problems. There remains little reason to believe that ellipsoid-based methods can compete with the simplex method.

In another example. numerical analysts at ORNL and Stanford, collaborating with colleagues at the University of Tennessee and the University of Waterloo. have developed a new method for solving very large sparse least squares problems. The method allows great flexibllity in partitioning very large problems and utilizing auxiliary storage, while avoiding numerical instabilities that plague current methods. Initial tests on problems with 20.000 equations in 5.000 unknowns indicate the method will be applicable to a wide variety of DOE problems. such as the analysis of the structural integrity of fission and fusion reactors and the analysis of the efficiency of combustion processes.

Numerical Methods for Partial Differential Equations play a dominant role in the modeling of energy systems and access to supercomputers and collaboration with teams of scientists or engineers are usually necessary to 
develop techniques that can be ultimately used in realistic DOE problems. Currently, this category emphasizes methods applicable to computational fluid dynamics. Although activities in Analytical and Numerical Methods are frequently pursued in collaboration with DOE mission programs (e.g. computer simulation of the combustion of fossil fuels). the research described here is definitely not intended to produce computer models. Rather it yields methodology and tools that can be used by DOE scientists and engineers to construct their models of specific phenomena. The principal objective is to develop methodology and tools applicable to currently intractable problems.

Computational fluid dynamics has made important practical contributions to aeronautical engineering. In particular. supercritical wings used in the last test generation of fuel efficient passenger and transport planes have benefited from extensive computer studies that enable design tradeoffs to be evaluated with speed and efficiency. Computer programs developed at the Courant Institute have played an important role in both the design and analysis of such wings. For example, a code for the design of three dimensional transonic swept wings has recently been developed. This code has been used in conjunction with the a major airframe design company to reduce the pressure gradients near the wing tips that are responsible for boundary layer separation. The code also provides useful estimates for the reduction in wave drag resulting from such improvements in design.

Computer modeling of turbulent combustion is an essential design tool in the effort to produce engines that use fuel efficiently and produce less pollutants. The Applied Mathematical Sciences program supports applied mathematicians at LBL. Sandia, and Courant who have developed algorithms that can provide practical computer models of the combustion process in realistic geometrles. This year for the first time a mathematical model that reproduces the experimental results of the combustion process in a cylinder was developed. These methods have also turned out to be very useful in the modeling of oil reservoirs and other areas of fluid mechanics. The recovery of oil from oil fields can be enhanced by the injection of water. dilute solutions. steam or gas. A computer model for these procedures. based on solving the governing partial differential equations, has been developed jointly at LBL and $a$ at the Courant Mathematics and Computing Laboratory. The model incorporates the relevant chemical and physical processes (condensation and phase transition) and fluid dynamical processes. including fronts of discontinuity. The model predicts realistic flow patterns. including the formation of instabilities that lower the amount of oil that can be recovered. The eventual use of the model is to determine by computer experiments the optimal procedure suitable for each field.

\section{Information Analysis Techniques}

In FY 1981. \$1.880.000 was allocated for Information Analysis Techniques. which seeks innovative approaches to the manipulation and analysis of large. complex collections of scientific, engineering. environmental, energy. and socio-economic data. It addresses questions basic to energy policy activities as well as the analysis of energy systems and their impacts. A new component of the AMS research program, it is organized into three categories: Statistical Methods. Data Management, and Display and Analysis Systems.

Statistical Methods addresses issues fundamental to analyzing both the input and the output of data intensive studles. Emphasis is on exploratory data analysis. experimental design. and sensitivity analysis applicable to large. 
complex data collections and multi-parameter models. where traditional statistical methods have proven inadequate.

Data Management encompasses computer science research underlying the organization and retrieval of large. complex data collections. Currently. the interface with interactive display and analysis of numerical data is emphasized: plans include the interface with computational models and new projects on the design of database machines for very large data sets.

Display and Analysis Systems studies methods "for making graphics. data management. and analysis tools more unified and easier to use. Initial efforts focus on the display and analysis of geo-coded data. New efforts are beginning to study techniques for displaying multidimensional sets representing large experimental data sets and the output of complex analytical models of combustion processes.

An example of a project involving all the categories of Information Analysis Techniques is being carried out by a collaboration of computer scientists and statisticians at two national laboratories and three universities (LBL. PNL. Princeton. Stanford. and George Washington). The primary areas of interest are exploratory data analysis of very large data sets using state of the art techniques involving color graphics and computer networks.

\section{Advanced Computing Concepts}

In FY 1981, \$3,100,000 was allocated for research in Advanced Computing Concepts. an activity that is based upon three premises: 1) the computer industry will continue to improve cost-performance ratios for hardware. 2) hardware systems will increasingly be tailored to specific classes of applications, and 3) the cost of software development and maintenance will dominate all other computing costs. Based upon these premises, three categories of Advanced Computer Concepts are distinguished: Software Engineering. Distributed Systems, and High Performance Systems.

Software Engineering includes research on programming languages and automated programming aids. with emphasis on ultimate applicability to large-scale computational models. Adopting a "bottom-up" approach. Software Engineering currently seeks a better understanding of the process of creating transportable. high-quality software implementations of algorithms for basic computational problems. the building blocks of computational models, and therefore interacts strongly with Computational Mathematics.

The most important effort in this area is a cooperative project called TOOLPACK. jointly funded by NSF. NASA, and DOE and including research teams from Argonne National Laboratory, the University of Colorado. the University of Arizona. Purdue. the Jet Propulsion Laboratory. the Numerical Algorithm Group in the UK, and IMSL. Inc. The purpose of the TOOLPACK project is to design and implement a software system for building. testing. and maintaining numerical software packages. The experience gained from the design and implementation of EISPACK. LINPACK. and others will be incorporated in the basic capabilities of TOOLPACK.

Distributed Systems includes research on both local and remote networks. Research on local networks emphasizes performance of links between worker computers (e.g.e supercomputers). mass-storage systems. and interactive access. Research on remote networks addresses the problem of resource utilization in a heterogeneous environment (e.g. program development on a highly interactive system with automated programming aids, program execution on a supercomputer. and analysis of results on an interactive graphics 
facility).

Several years ago the Applied Mathematical Sciences program sponsored an investigation of computer networks as a technique for resource sharing among DOE laboratories. The principal finding was that the main barrier to resource sharing had little to do with networks per se. The problem was the necessity of learning many new operating systems. command languages. and different styles of user interfaces once network access had been accomplished. To attack this problem, the Advanced Systems group at LBL began developing a Virtual Operating System that would provide a uniform user environment on any computer system. Over a period of two years. this project generated a lot of enthusiasm in the research community - in fact. a Software Tools Users Group was organized that now claims over 1000 members. In the process of investigating the principles of virtual operating systems. the LBL group produced a practical and useful set of software tools that has been implemented on over 25 different computer systems. Both industry and other government agencies have been eager to assimilate the practical aspects of this research. and the results have been published in the Communications of the ACM. Current work is aimed at developing interprocess communication primitives that will allow heterogeneous computer networks to behave as a single, integrated computational resource.

High Performance Systems responds to the fact that computer hardware development has reached a fundamental limit imposed by finite signal propagation speeds. While new technologies may alleviate heat generation in circuitry and therefore permit more compact and thus faster systems. increased computing power will ultimately depend upon parallel execution of instructions. High Performance Systems. therefore. emphasizes two critical areas: the match between algorithmic structure of large-scale computational models and the possible architectural structure of future supercomputers. and the transportability of applications software between successive generations of supercomputers. Several activities in Advanced Computer Concepts are pursued jointly with the NSF's Computer Science Research Program and the DOD DARPA's Information Processing Techniques Research Program. For example. NSF is supporting investigations of fundamental changes in computer architectures and DARPA is supporting related implications of VLSI technology. while DOE supports their impact on the solution of partial differential equations describing energy systems on the supercomputers of the future.

At the level of electronic components. the art of computer building is advancing very rapidly. Single, fingernail-sized electronic "chips" are already as powerful as yesterday's minicomputers, and by the end of this decade very small computers, each as powerful as some of todays largest. should become available. This technological progress is bound to trigger major changes in the way computer systems are designed and used. A particularly exciting possibility is to combine thousands or tens of thousands of these powerful one-chip computers into "ultracomputers" capable of computing hundreds of times faster than is now feasible. This would allow computational study of many important chemical, meteorological, physical. and blological models that presently lie out of reach. A project to study supercomputer designs and programming strategies is being supported at the Courant Institute of Mathematical Sciences of New. York University. M.I.T.. the 
University of Illinois. the University of Texas. UCLA. LLNL, and LANL.

\section{Special Projects}

In FY 1981. \$500.000 was allocated for Special Projects to support conferences and working groups to evaluate program directions: liaison activities to stimulate interactions between AMS researchers and DOE computer users: and selected special projects outside the previously defined topic areas. Examples of these activities include a High Speed Computer Workshop sponsored by LLNL and LANL that includes representatives from all the major mainframe manufacturers and scientists from the DOE community, the Berkeley Workshop on Distributed Data Management and Computer Networks (the Fifth was held in 1981), the Adaptive Mesh Workshop at LANL's Center for Non-Linear Studies, and several visiting scientist programs that serve to bring distinguished visitors from the universities to the national laboratories.

\section{Capital Equipment}

In FY 1981 \$706.000 was allocated for capital equipment in Applled Mathematical Sciences for the DOE laboratories and NYU. Equipment funds for university programs do not come from this budget. The Applied Mathematical Sciences research program is supporting the development and operation of Research Comquting Facilities (RCF's) at several DOE National Laboratories and universities. Each RCF will consist of one or more minicomputers dedicated primarily to a specific thrust of the AMS research program. Although the principal users of an RCF will be the staff at the host site. each RCF will be available as a resource for other AMS researchers participating in the associated thrust area. The RCF's will be linked by one or more existing computer networks to:

a. facilitate access by researchers without a local RCF:

b. stimulate interactions among various AMS supported research groups: and

c. provide a resource for research on distributed systems.

Where appropriate, an RCF will be linked to the Central Computing Facility of the site. Inus, the HCL's will also serve as gateways to large-scale scientific computers and other DOE computing resources.

The establishment of Research Computing Facilities will provide mathematicians. statisticians, and computer scientists with the resources to explore innovative new approaches to utilizing the developments of computer technology. while simultaneously developing new technological frontiers. While other scientists and engineers use the computer as a tool to simulate and analyze experiments, the computer research community uses the computer itself as the object of experimentation. This experimentation is obviously incompatible with the stability of the computing environment required to serve the computing needs of other disciplines. The establishment of RCF's is consistent with the recommendations of a select panel of academic and Industrial representatives reported in "Rejuvenating Experimental Computer Science: A Report to the National Science Foundation and Others". The formulation and implementation of plans are, therefore, being coordinated with NSF and DARPA. Essentially all FY 1981 Capital Equipment funds will be allocated to these Research Computing Facilities. 
AMES LABORATORY

Department of Mathematics and

Applied Mathematical Sciences

lowa State University

Ames, lowa 500?1

01. ANALYTICAL AND NUMERICAL METHODS

$\$ 225.000$

01

James Corones. B. Carlson. M. Davison.

A. K. Gautesen. H. McMaken. B. defacio.

R. Krueger

\section{Applied Analysis}

The work being done is exclusively in the area of applied analysis. The effort is focused on basic and applied problems in linear and nonlinear wave propagation. In the area of linear wave propagation. particular attention is given to direct and inverse scattering theory and related areas. $e$. g.. tomography. The purpose of this work is to develop mathematical procedures and tools that can be used to characterize and/or image nonuniformities in materials (density variations. stress fields, cracks, poiential, for example). Two types of approaches are pursued: 1) direct scattering. in which the nonuniformities are given and the objective is to compute the scattered field. e. g.. of elastic waves scattered by cracks. and thereby determine characteristic signatures of scatterers: and 2) inversion procedures, in which incoming and outgoing signals are assumed known and the objective is to deduce the nonuniformities from this knowledge. $e$. 9 .. mapping density variations using $x$-ray tomography. Inversion studies attempt to incorporate realistic constraints such as limited angles of view. limited apertures and noise. The techniques developed have wide, application. including quantitative NDE. geoprospecting, remote sensing of nuclear waste. plasma diagnostics, and fission reactor diagnostics. At present, QNDE is the major applications orientation of the effort. There is a modest effort in nonlinear wave propagation focused on the study of equations that support soliton solutions. In addition. work on special functions directed at facilitating their use and computation is carried out. 
ARGONNE NATIONAL LABORATORY

Applied Mäthematics Division

9700 South Cass Aveniue

Argonne. Illinois 60439

02. ANALYTICAL AND NUMERICAL METHODS

$\$ 800.000$

01

R. J. Royston. P. C. Messina.

H. G. Kaper, G. K. Leaf.

B. J. Matkowsky. W. J. Cody. Jr.

J. M. Cook. J. J. Dongarra.

B. S. Garbow. K. E. Hillstrom.

J. N. Lyness. M. Minkoff. J. J. More'

D. C. Sorensen. T. F. Coleman

This task consists of research in the areas of applied analysis and computational mathematics. Current interest in applied analysis centers on the modeling and analysis of reaction-diffusion systems, bifurcation phenomena in combustion problems. and fluid flow problems related to loss-of-coolant analysis in reactor engineering. While specific areas of investigation may vary in response to changing DOE needs. the long-term objectives remain the same: the mathematical modeling of observable phenomena, the analysis of the mathematical model, and the interpretation of the analytical results. The computational mathematics research involves the design and analysis of numerical algorithms. the development of special techniques to measure .algorithm reliability and efficiency. and the preparation of software based on broadly applicable computational methods. Efforts focus on development of new algorithms for Bessel functions. investigation of optimization algorithms. design and dissemination of quadrature techniques. study of methods for solving partial differential equations. and development of improved techniques for solving problems in numerical linear algebra. In addition, work on software evaluation has been renewed. and special methods are being developed for use in systems modeling and chemical equilibrium problems.

03. ADVANCED COMPUTER CONCEPTS

$\$ 720.000$

03

R. J. Royston. P. C. Messina.

J. M. Boyle. W. J. Cody. Jr.,

W. R. Cowell. J. J. Dongarra.

K. W. Drilz. J. R. Gabrlel.

B. S. Garbow, K. E. Hillstrom.

J. J. More'. B. T. Smith.

D. C. Sorensen. L. T. Wos.

B. W. Char. E. L. Lusk.

R. L. Veröfi. S. K. Wiriker

This task consists of research in the area of software engineering, with the objective of producing highly reliable. easily usable computer software at reduced cost. Activities range from work on program transformation and verification to language-related studies on extensions to Fortran and PL/I. Other topics of interest include coordination of a multi-institutional project to produce tools for numerically oriented software and participation in a project on computer-assisted nuclear power operation. In addition, a short-term investigation has been initiated on the use of computer algebra systems for numerical computation. 
04. SPECIAL PROJECTS/ RESEARCH COMPUTING FACILITY

A. J. Royston. P. C. Messina.

J. J. Dongarra. G. Roediger

This special project involves the development and operation of a Research Computing Facility. The facility is intended to provide an opporturity to explore the effectiveness of using smaller computers for mathematical software research and for collaborative studies among AMS-funded groups. The RCF is currently based on a Digital Equipment Corporation VAX 11/780 computer with three disk drives and one tape drive. Experiments on the RCF are already under way in computational mathematics. software evaluation. and algebraic manipulation. In addition. work is progressing on the development of a new automated reasoning system and a new version of the TAMPR transformation program. 
BROOKHAVEN NATIONAL LABORATORY

Appllied Mathematics Department

Upton. New York 11973

05. ANALYTICAL AND NUMERICAL METHODS

C. Goldstein. H. Stewart. J. Pasciak. J. Ho

$\$ 275,000$

01

The emphasis of this research is on the numerical solution of partial differential equations and their application to problems of importance in energy research. The work involves both development of new methods to improve techniques for existing problems and also the extension of existing techniques towards more complex problems. especially those which are intrinsically three dimensional. Some effort is also devoted to research in image reconstruction and in optimisation. Finite element methods for unbounded domains are being developed. These problems arise in connection with fluid flow around objects and in the propagation of acoustic and electromagnetic waves. The methods introduce a finite but distant boundary and a graded mesh. Iterative techniques for solving the resulting equations are being implemented as practical codes. In connection with scattering of waves in the presence of a disturbance. careful consideration of the radiation conditions and iterative finite element procedures lead to an efficient algorithm for solution which is being implemented and tested. The reliable computation of magnetostatic fields in three dimensions. of considerable importance in many research areas where sophisticated magnets are needed. is being pursued through both integral equation methods and finite element methods using Lagrange multiplier techniques to treat the interface condition. Research in multiple fluid flow calculations is being pursued with particular emphasis on modelling of nuclear reactors. By generalizing a combination of new fractional time step methods and a new convergence acceleration technlque it is hoped to make considerable progress towards overcoming all time step limitations. Mathematical and numerical problems of image reconstruction in two and three dimensions are being investigated.

06. INFORMATION ANALYSIS TECHNIQUES $\$ 225.000$

02

C. S. Kao. H. Robbins, J. van Ryzin. J. Ho

Statistical Methods

Research under this heading is primarliy involved with the development and application of statistical methods and analysis. Many of the applications at BNL are focused on blostatistical problems in collaboration with the environmenatl and medical programs, but the emphasis is on methods which have broad applications. Work in survival analysis is involves the handling of longitudinal survival data with censoring due to incomplete followup in the presence of covariates of interest. Potential applications are to many fallure time and reliability studies. Particular applications are being made to assessment of health offects of energy related ponllutants and in criterla for a proposed neutron capture therapy trial for brain tumors. Research on risk assessment includes modelling and estimation in the area of dose response data with and without time-to-response as a factor. These techniques are bing used in the course of participation in a National Academy of Sciences panel on nitrites. Empirical Bayes methods are of growing importance. Comparative studies of linear and general Empirical 
Bayes methods and of and adaptive generalization are being carried out. The problem of Empirical Bayes techniques for simultaneous estimation of many variances is under investigation. Work on sequential analysis is focused on the development of sequential selection rules for choosing the best of a set of binomial populations. This has important applications to clinical trials, but also to a wide variety of testing and evaluation problems where the sampling or testing costs are high. Other research includes multivariate analysis and holistic preference evaluation. 
CALIFORNIA INSTITUTE OF TECHNOLOGY

Department of Applied Mathematics

Pasadena, California 91125

07. ANALYTICAL AND NUMERICAL METHODS

$\$ 162,800$

01

H. B. Keller. P. G. Saffman

Numerical Analysis. Computing, and Fundamental

Studies of Energy and Máss Transfer

Studies and investigations are being conducted in the development of mathematical and computational techniques for analysis of a variety of nonlinear problems. mainiy in fluid dynamics. which are concerned with phenomena involved in scientific and technological problems related to energy production and utilization. The objective is to improve existing techniques. develop new mathematical and computational techniques. and increase understanding of the physical and engineering phenomena. Improved methods have been developed for the calculation of bifurcation and instability phenomena and they have been applied to rotating fluids, pipe flow. and water waves. New numerical methods have been devised and tested on the driven cavity. An explanation has been found for the observed widths of fingers in two dimensional simulations. 
THE GEORGE WASHINGTON UNIVERSITY

Department off Electrical Engineering and Computer Sciences Washington, D.C. 20052

08. INFORMATION ANALYSIS TECHNIQUES

$\$ 0($ in FY 1987) 02 Michael

B. Feldman. James

D. Foley

Research on Information Display Systems

Several important aspects of Information Display Systems are being studied. The main objectives of the project are:

1. To develop methodologies for separating the creation of application system modules from the creations of user "front ends". through the definition of a "funnel" or "abstract interaction handier" through which all interaction related information passes to and from the user:

2. to further develop and refine methodologies for the formal specification of user command interfaces and the computer aided evaluation of these interfaces with respect to human factors attributes:

3. to use and evaluate a set of computer graphics abstractions which has been proposed as a standard. to determine whether these abstractions are workable as defined or whether they require modification.

We are also distributing on request the GWU Core Graphics package. 
KENT STATE UNIVERSITY

Department of Mathematics

Kent. Ohio 44242

09. ANALYTICAL AND NUMERICAL METHODS

$\$ 185,000$

01

R. S. Varga. P. S. Wang

\section{Applications of Numerical Analysis and \\ Computer Symbolic Computation}

The major emphasis for this research is on the theoretical basis for workable numerical algorithms for general iterative methods in linear algebra. and for workable algorithms for inclusion in vaxima. The research areas are:

1. An asymptotic analysis of basic iterative methods to matrix equations arising from finite element methods.

2. The use of symbolic and numerical techniques to attempt to settle the long standing Whittaker conjecture in function theory.

3. The integration of symbolic (exact) and numerical (approximate) computing techniques.

Current emphasis is on the theoretical understanding of matrix factorization. incomplete polynomials, and generalzed Bessel polynomials. Many improvements to the Vaxima system will be made in the area of polynomial factorization algorithms, partial fractions. complex numbers, and Pade' approximates. 
LA JOLLA INSTITUTE

Center for Studies of Nonlinear Dynamics

P. O. Box 1434

La Jolla. California 92038

10. ANALYTICAL AND NUMERICAL METHODS

$\$ 97.000$

01 Kenneth Watson

\section{Studies of Turbulence in Dynamical Systems}

We are applying a numerical method based on Taylor series expansions to uncover the analytic structure (singularities) of several dynamical systems that display "turbulent" behavior. Among these are the Lorenz. HenonHeiles, and point-vortex systems. Our results show a correlation between the structure of the complex-time singularities and the qualitative properties of the real-time motion.

By considering the detailed properties of the expansion of a solution about a singularity. we have been able to predict. for a given system. the nature of its singular set. Natural boundaries with a "fractal" structure are found for a wide of class of non-integrable dynamical systems. Our analysis agrees well with. and was guided by. the direct numerical continuation by Taylor series of these systems to the complex time domain. In addition. our results show the precise role of the multivaluedness of the system's singularities in determining its non-integrability. This is the complement to the known correlation between single-valued integrals (Painleve' property) and integrability in dynamical systems, that was first formulated by $S$. Kowalevsky in 1890.

Additionally, we are examining the extension to a complex variable of certain mappings of the real line whose iterates are known to exhibit chaotic behavior. Taylor series methods are being developed for nonlinear partial differential equations.

Finally. we are studying the "scattering" propertles of point-vortex systems. These systems. which provide a useful model of two-dimensional hydrodynamics, are known. in general. to be non-integrable and. by calculation. to be chaotic. Our study focuses on the scattering dynamics of point vortex pairs. 
LOS ALAMOS NATIONAL LABORATORY

Theoretical Division

University of California

P. O. Box 1663

Los Alamos. New Mexico 87545

11. ANALYTICAL AND NUMERICAL METHODS

$\$ 287,500$

01

Basil Nichols. J. E. Dendy. Jr.

D. Butler, J. M. Hyman

Numerical Analysis and Nonlinear Mathematics of Energy Systems

The future effectiveness of many physics and engineering design codes important to the national interest depends upon developing improved numerical and analytical methods for large systems of partial differential equations (P. D. E.). Such systems, modelling complex hydrodynamic flows (multidimensional. compressible, and reactive) are the focus of the two complementary activities in the present task. The first is to improve the reliability of such predictive computational models by unraveling the analytical structure of the corresponding nonlinear P.D.E. With the help of rerent constructive nonlinear analysis techniques. this activity emphasizes the structural properties (multiplicity, global stability, stiffness) of the nonlinear waves that arise from the interaction of chemical processes, multiple phases and fluid flow fields. The second activity is to derive correlated, highly efficient numerical algorithms for these systems, especially as to the integration of large dispersive systems. adaptive mesh refinement techniques. the approximation of boundary conditions and the structured design of production codes.

This task is carried out in close cooperation with the Center for Nonlinear Studies at Los Alamos, including the joint organization of a workshop on Numerical Adaptive Mesh Methods.

The final task in multidimensional numerical fluid dynamics provides a means of making many recent advances in large scale computational fluid dynamics available for use in DOE programs, where such theoretical analysis was previously thought intractable. This support enables the development and documentation of user-oriented. publicly available computer codes.

\author{
12. ADVANCED COMPUTER CONCEPTS \\ $\$ 425,000$ \\ 03 \\ Bllly Buzbee. Thomas Jordan. \\ Paul Frederickson. Robert Hiromoto. \\ Joseph Fasel. Ingrid Bucher
}

High Performance Systems

By 1985. venoors of high performance systems may offer multiprocessor systems in which four or more processors share a common memory and collaborate on a common task.' Such a system could be used to multiprocess a numerical simulation. but to do so requires revolutionary changes in scientific computing. In particular, we will need algorithms that lend themselves to efficient partitioning across processors and software that facilitates implementation of these algorithms. These are the objectives of Los Alamos' research in high performance systems.

Although multiprocessing is an old idea. there is little experience with it. Thus in addition to developing algorithms. we have set as one of our goals 
the performance of computational experiments using any suitable equipment. Experiments are necessary to 1) determine if our algorithms work, and 2) if so. how well they work - l.e.. obtain quantitative performance datá. The latter is particularly important because overall performance of a multiprocessor system is strongly dependent on average utllization of individual processors. Since full scale DOE simulation models often require several person-years of effort to transport from one system to another. our technical approach is to incorporate our algorithms into "benchmark models" that are representative of full scale simulations but of manageable proportions. Benchmark models associated with particle-in-cell calculations. Monte Carlo calculations, and diffusion calculations will be developed and used in our experiments. These three areas encompass a large portion of DOE large scale calculations in energy research and weapon design.

Based on the experience obtained from these experiments, we will explore language constructs for implementing our aigorithms. 
LAWRENCE BERKELEY LABORATORY

Physics. Computer Science and

Mathematics Division

University of Callfornia

Berkeley, California 94720

13. ANALYTICAL AND NUMERICAL METHODS

$\$ 325.000$

01

A. Chorin. P. Concus. F. A. Grunbaum

The Analytical and Numerical Methods program at LBL encompasses the areas of applied analysis. computational mathematics. aod numerical muthods for partial differential equations. The main thrust is the development of analytical and numerical techniques for analyzing turbulent combustion phenomena. such as occur in an internal combustion engine. and multiphase porous flow phenomena. such as occur in petroleum or geothermal reservolrs. Computational techniques for obtaining solutions that are discontinuous or nearly discontinuous for hyperbolic and parabolic partial differenthal equations are unique in that they use sampling procedures and "dictionaries" of known phenomena as building blocks in the numerical schemes. The goal is the construction of realistic models that are suitable for comparison with experiments and are capable of being used for engineering purposes. Dynamic representation of the numerical results. that is the application of interactive computer graphics. offers a highly effective environment and tools for understanding of the models and computational results. Other topics of primary interest include the development of methods for the numerical solution of elliptic partial differential equations. the analysis of particular classes of equations arising in the study of caplllary phenomena and the development of methods for the reconstruction of images of objects from projection data of their transverse sections.

\section{INFORMATION ANALYSIS TECHNIQUES $\$ \$ 450.000 \quad 02$ \\ C. Quong. A. Shoshani. \\ H. Holmes. W. Greiman}

Research in Information Analysis Techniques seeks innovative approaches to the manipulation, analysis, and display of large, "complex data sets. focussing on enhancement of the end user's perception of the computer as an analysis tool. Data management research is directed at creating efficient algorithms for data storage and access. developing data models appropriate for large. statistical data bases, and query language design. The major thrust of research in display techniques is the development of interactive graphics for visualizing data as part of the analytical process as well as for the design of presentation displays. The use of color. shading. text, symbols, arid the deslgn of a proper user interface are all important factors in producing optimal graphical displays that will maximize communication of information to the viewer. LBL's Socio-Economic Environmental Demographic Information System (SEEDIS) provides a framework for development, experimentation. and demonstration of information analysis techniques. 
15. ADVANCED COMPUTER CONCEPTS

03

Dennis Hall. Joseph Sventek

Advenced Computer Concepts is primarily concerned with understanding the integration and engineering of computing "systems," with particular attention to what will be possible five years hence. The effort may be characterized as applied computer science research. bridging the gap between purely theoretical computer science and the future operational needs of DOE computing. The approach is interdisciplinary in the areas of software engineering. distributed systems, and performance evaluation.

In software engineering. initial effort has focused on providing a stable software development environment for research. The virtual operating system approach has been embraced by governmental, industrial and academic groups as a demonstrated method to improve the productivity of people building software. With this providing the foundation. problems in software architecture and software management are now being explored.

In distributed systems, the utillty of long-haul research networks is now established. but the rapidly evolving local network technologies are only begining to be understood. Research is underway in communications processing. interprocess communication techniques, protocol design. and hardware interconnection techniques.

Performance evaluation effort is concerned with understanding file storage systems and developing algorithms which expedite their efficient use. This research will significantly impact design of new. high-performance storage hierarchies.

16. SPECIAL PROJECTS/

$\$ 232.500$

04

RESEARCH COMPUTING FACILITY

C. Quong. M. O'Dell

The Research Computing Facility (RCF) provides the computational resources needed to support the ongoing basic research in Applied Mathematical Sciences. It serves as a testbed for the evaluation and deployment of new computing capabilities as well as serving as the hub of the Electronic Mail System. Maintenance and development of the RCF. Including integration and evaluation of emerging computing technologies. is a focal point for collaborative interaction with other $D O E$ research sites and with researchers in computer science throughout the university and industrial communities.

Liaison activities with DOE staff managing the Applied Mathematical Sciences research program were maintained this year as a one-time task. 


\author{
LAWRENCE LIVERMORE NATIONAL LABORATORY \\ Computer Sclence and Mathematics \\ University of California \\ Livermore, California 94550
}

17. ANALYTICAL AND NUMERICAL METHODS $\$ 175.000$

01

George Michael. Fred Fritsch. Alan Hindmarsh

\title{
Computational Mathematics
}

This is one of three DOE. OBES-funded project areas at LLNL, within the overall goal of more effective high-speed. large-scale computational capabilities. The computational: mathematics task addresses issues of numerical methods and software design in (A) ordinary differential equation initial value approximation and $(B)$ interpolation and approximation problems. in ODE's. the new general-purpose solvers LSOOE and LSODI have proved quite successful and are widely. used. A variant for general sparse Jacoblans was written with $\wedge$. Shorman. and another. which does automatic methodswitching was written with L.Petzold. Other useful variants are planned, and all these solvers will form a systematized collection called ODEPACK. Testing and documentation work emphasizes applications to partial differential equations.

Work continued on methods and software for numerical approximation. A paper describing an algorithm for monotonicity-preserving bicubic interpolation has been submitted for publication. and good progress has been made in developing software that implements the algorithm. The resulting surface is free of the unphysical 'bumps' and interpolate equation of state data. PCHIP. a comprehensive portable package of one-dimensional interpolation software, is nearly complete. Work has begun on extending these ideas to area- and shape-preserving histogram approximations, which are needed in many numerical simulation studies.

18. ADVANCED COMPUTER CONCEPTS George Michael, Garry Rodrigue. James McGraw

\section{High Performance Systems}

This category encompasses work in the second and third of the three DoE. Office of Basic Energy Sciences funded projects in mathematics and computer research at LLNL. All three projects contribute to the single goal of developing more effective high speed. large scale computers and methods for their use. The manner of working toward this goal involves close interaction with other DOE laboratories, universities, and computer manufacturers. The first of a planned eeries of meetings with these groups was held at the start of April. 1981. The meetings are by invitation only and their purpose is to acquaint the groups with the anatomy of LLNL and LANL computational problems.

The first of the two projects mentioned above studies languages that can exploit parallel architectures. the most likely form of super-speed computers in the near future. At the same time these language developments are being so designed that they can be applied to more radical computers expected late in this decade. Work is going on also in major additions to existing languages. The data flow language $V A L$ now has a syntax-directed editor attached to the compiler that was started last year. It will be tested 
on our current large computer systems. - The VAL language itself is being extended as well features needed to complete it.

The last project is based on a restructuring of the basic computer models used to define the large physics problems being worked on at LLNL. The idea is to certify that the numerical algorithms fit, as well as possible, the computer structures of the future. Work in these numerical algorithms centers on those for solving partial differential equations. Algorithms based on local rather than global phenomena are being developed. So far this year a highly parallel variant of the conjugate gradient method was developed for solving linear systems arising from elliptic. PDE's. A predictor-corrector-iterative method for solving implicit parabolic PDE's on parallel processors is currently being studied. An adaptive mesh refinement method for solving hyperbolic PDE's has been developed and is currently being analyzed for $1-D$ systems. Extensions to $2-D$ are now also being started. 
MASSACHUSETTS INSITUTE OF TECHNOLOGY

Laboratory for Computer Science

545 Technology Square

Cambridge, Massachusetts 02139

\section{ADVANCED COMPUTING CONCEPTS} Jack B. Dennis

\section{Data Flow Architectures}

The final year of this three year research program on data flow architecture for high performance computer systems is devoted to the two primary areas where questions remain regarding the prospects for successful construction of practical systems: machine code generation and structuring algorithms that achieve effective and balanced use of the processing elements and memories of a data flow computer: and. hardware design studies that permit analysis of the effort and cost of machine construction.

The programming language VAL, our user programming language for data flow computation. Is the source language for our work on translating data flow programs. Our emulation facility for evaluating proposed data flow archltectures will serve as the target machine. We can now compile small programs in VAL into data flow machine language and run the machine code to produce numerical results. The main focus is now on how to structure computations involving large volumes of array formatted data. This work is guided by analysis of several large benchmark computations provided by sponsors.

We expect to complete specification of a practical large scale data flow supercomputer during the third year of support. 
NORTHWESTERN UNIVERSITY

Department of Engineering Sciences and Applied Mathematics

Evanston. Illinois 60201

20. ANALYTICAL AND NUMERICAL METHODS $\$ 73.000$

01

Bernard J. Matkowsky

Research in Nonlinear Problems of Energy

This research continues theoretical investigations of bifurcation and nonlinear stability theories with emphasis on applications to combustion and flame propagation. Among the topics being examined the principal ones are: 1) formulation of cellular flame tronts: 2) effect of acceleration on the stability or propagating flames: 3) propagation of pulsating reaction fronts in solid fuel combustion: and 4) response of a system exhibiting bifurcation to small inhomogeneities. 
NEW YORK UNIVERSITY

Courant Institute for Mathematical Sclences

New York. New York

21. ANALYTICAL AND NUMERICAL METHODS

$\$ 1,300,000$

01

Peter D. Lax. R. Burridge

O. Betancourt. O. McBryan

O. Widlund. P. Whitlock

E. Isaacson. M. Overton

M. Kalos. H. Strauss

R. DiPerna. M. Luskin

\section{CALCULATION OF FLOWS WITH SHOCKS}

The aim of this research is to develop effective numerical methods for solving the equation of compressible gas flow. and equations like it. and then to apply thern to the calculation of flows with shocks. The proposed class of schemes yerieralizes a mothod developed by Godunov, and one developed by Glimm. The novel idea is to replace the exact solution of the Riemann problem. a basic ingredient in both of the above mentioned methods. by approximate solutions. We have shown this strategy works, and works well. provided that the approximate Riemann solver satisfies the conservation laws whose solutions we are seeking, and satisfles the entropy inequality as well.

We are extending these methods to two-dimensional problems. using the method of fractional steps. Our preliminary analysis shows that such composite schemes always satisfy the conservation laws and the entropy inequality. However. the two-dimensional version of the schieme resolve 3 discontinuities less sharply. on account of the inability of one-dimensional sweeps to recognize a sharp discontinuity that propagates in an oblique direction. We plan to correct this by adding a single two-dimensional entropy check in place of two one-dimensional ones. We have clarified the validity of the entropy condition in two dimensions. and hope to devise schemes that will provide high resolution in two-dimenslonal problems.

P. D. Lax

\section{SHOCK TRACKING METHODS}

Many important physical processes involve moving shock fronts or sharp materlat interfaces. Thic project aims at developing computational methods for studying these phenomena. Applications will be made to a variety of systems encountered in energy development, including secondary and tertiary oil recovery simulation and the dynamics of gases and flame fronts.

Currently. a general purpose computer code. is under development for solving problems with shock fronts. It uses a shock tracking method and is capable of providing very high resolution at shock fronts. Even unstable fronts which cvolve into many fingers can be tracked. There will be no restriction on the number of physical phases or interfaces in the systems being studied. Development codes will be written in a higher-level language (c), which we have found to greatly increase productivity over using FORTRAN.

O. McBryan 


\section{MHD EQUILIBRIUM AND STABILITY}

We have developed a computational method to analyze equilibrium and stability problems in magnetohydrodynamics. Our ideas have". been implemented in several general purpose codes which by now are sophisticated enough to provide a very useful tool in the design of future experiments.

An important new direction is the development of a code to study threedimensional diffusion problems. This project is underway as a cooperative effort with $H$. Grad and A. Bayliss of the Courant MHD Division. Another new area is the study of $3-D$ perturbations in Tokamaks. examination of the assumption of nested surfaces. and island formation.

\section{O. Betancourt}

\section{MAGNETOHYDRODYNAMIC THEORY ?}

The purpose of this research is to gain understanding of the stability of conducting fluids and plasmas in magnetic fields. In particular. attention has been given to "ballooning", or pressure-driven instabilities, and tearing modes. which involve magnetic field line reconnection. Both analytic and numerical approaches are taken. Nonlinear. three-dimensional equations of motion. based on orderings in parameters such as toroidal aspect ratio. have been derived for tokamaks and stellarators and solved numerically. A new set of reduced equations has been obtained for mirror machines. such as Livermore TMX. The research results can be applied to understanding plasma physics experiments performed for DOE. and may also be used to predict the effects of substantially raising the plasma pressure in such devices, as would be necessary for commercially viable fusion reactors.

\section{H. R. Strauss}

\section{METHODS FOR NONSMOOTH OPTIMIZATION PROBLEMS}

Our main interest is that of designing numerical methods for solving nonsmooth optimization problems. These problems include the following: minimizing the maximum of a finite number of smooth functions (the minimax problem). minimizing the sum of absolute values of smooth scalar-valued functions (the $j 1$ problem). minimizing the sum of Euclidean norms of smooth vector-valued functions (the sum of norms problem), and others.

The minimax problem often arises in game theory and circuit design. while solving the 11 problem is useful in the robust analysis of experimental data. The sum of norms problem arises in many energy and economics applications, where sums of distances must be minimized. It also arises in many physical applications. including the discretized minimal surface problem. It can be shown that the minimax and 11 problems are equivalent to certain smooth constrained optimization problems, and so the wide body of knowledge associated with constrained optimization can be applied to obtain their solution. However, these constrained optimization formulations have a very special structure, and numerical methods must take advantage of this fact in order to be efficient. The sum of norms problem. on the other hand. does not have a computationally useful constrained optimization formulation: it is necessary 
to apply methods directly to the original problem.

The goal in all cases is three-fold: to provide an improved mathematical framework, efficient and reliable numerical methods. and convenient general-purpose software.

TWO STAGE ITERATIVE PROCEDURES FOR SOLVING LINEAR EQUATIONS

In many applications one wishes to solve the linear system of equations $A x=b$ by an iterative method. i.e.. generating $x(k), k=0.1 .2 \ldots$. where $x(k)$ converges to the solution $x$ as $k$ approaches infinity. Many iterative methods split the matrix $A$ into two parts, i.e.. $A=M+N$. and require at the $k$ th stage of the "outer" iteration that the system $\mathrm{Mz}(k)=$ $b$ - $A x(k-1)$ be solved for $z(k)$. Frequently, it is also desirable to solve these systems for $z(k)$ by an "inner" iterative procedure. We are interested in investigating how the convergence of the outer iteration depends on the accuracy required for each inner iteration. This technique has particular relevance to solving the large systems of equations arising from the discretizations of nonseparable elliptic partial differential equations.

M. Overton

COMPUTATIONAL MATHEMATICS -- NUMERICAL ANALYSIS

Work will continue on efficient methods of solving highly structured finite-difference and finite-element systems of equations. Capacitance matrix methods will be further developed for certain special. Dut important elliptic problems on general regions in two and three dimensions. We aim at providing an improved mathematical framework. fast and rellable methods, and convenient transportable FORTRAN programs. In this connection. the necessary new fast solvers for problems on simple reglons and with uniform meshes will be developed.

Further work will be done on iterative methods of conjugate gradient type. in particular to explore their use as acceleration procedures for solving preconditioned finite-element and finite- difference problems. Of particular interest are methods for problems which are not of positive definite symmetric type. Variants of Brayden's methods appear to be quite promising for such cases.

These tools will be explored for the numerical solution of the stationary and the time dependent Navier-Stokes equation and other important applications. An attempt will be made to develop reliable methods for general regions. possibly with an order of accuracy higher than two. The aim of this research is to retain as much as possible the simplicity and economy of the finite difference methods which have primarily been developed for rectangular regions.

In this work. special attention is paid to efficiency of implementation and the quality of the software, since these methods are heavily used as subprograms when solving more complicated partial differential equations related to a wide variety of computational physics problems. (Early work on these problems is being carried out jointly with Petter Bjorstad.)

O. Widlund 


\section{CLIMATOLOGY AND METEOROLOGY}

Assuming the likelihood that increased levels of carbon dioxide dust and other pollutants in the atmosphere may produce an irreversible change in the climate constitutes an important problem. In this connection. we have been formulating mathematical models in which we can study quantitatively how and why ice caps form or desert ages might develop. Such studies require the design of numerical methods that are accurate for long term calculation. This is a major aspect of our work.

We are developing methods to improve the accuracy of weather forecasts. We are finding efficient ways to incorporate observations made by space satellites into the standard numerical procedures for predicting the weather.

We are studying how to use an ultracomputer, (a parallel assemblage of thousands of CDC 6600-like computers) in the large scale weather forecasting problem. Here our work may influence the design of such ultracomputers.

E. Isaacson

\section{THEORY AND APPROXIMATE CALCULATIONS OF SHOCK WAVES}

Our research has been concerned with the approximation of solutions to nonlinear differential equations. Specifically. we have dealt with hyperbolic systems of conservation laws arising in continuum mechanics and corresponding solutions containing shock waves. We have investigated the problem of convergence of the approximate solutions generated by several different methods, e.g. classical finite difference schemes that are conservative in the sense of Lax and Wendroff and parabolic systems modeling diffusion and viscosity. For a class of methods of this type, we have shown that if the Lax entropy condition is satisfied and if the amplitude of the approximations is uniformly bounded then one obtains convergence.

\section{R. J. DiPerna}

\section{FLOW IN PIPES}

The work deals with the flow of compressible fluids in a pipe whose wall exerts a damping force. Without damping the formation of shocks is inevitable: this study shows that damping by wall friction can prevent shock formation, and obtains quantitative estimates for this to be the case. Results of this kind are of use in analyzing the flow of gas pumped through a pipeline.

M. Luskin

\section{COMPUTATIONAL MODELING -- METHODS IN MATERIAL SCIENCE I}

This research deals primarlly with the properties of matter that is not in thermodynamic equiliorium. In solid systems we are principally concerned with the development of grains in alloy quenched from the melt. This is of greal importance since the metallurgical properties are determined in large part by the imperfections that develop. Two kinds of 
phenomena seem to be involved. One is classical nucleation and growth in which clusters form and grow at the expense of smaller clusters and isolated atoms. Another is "spinodal decomposition" in which random composition is unstable against the formation of macroscopic zones of different compositions. In the liquid vapor system. nucleation -- usually with the help of impurity nuclei -- is responsible for the formation of rain and aerosol droplets.

Our primary tool of investigation is computer simulation of atomic models with some form of dynamics that drives the system toward equilibrium. Of necessity. the thermodynamics of these systems must be investigated when they are not adequately known. For alloy systems, we use Kawasaki kinetics in which adjacent atoms are exchanged when such a change is energetically favorable. For fluid systems. specific atomic force laws are used along with molecular dynamics as an algorithm.

Applications. Simulations of atomic systems aid in the understanding of the metallurgical properties of alloys. The study of nucleation leads to greater understanding of the formation of aerosols.

M. H. Kalos et al.

COMPUTATIONAL MODELING -- METHODS IN MATERIAL SCIENCE ॥

Polymer Systems

We study the behavior of individual polymers and of systems of polymeric molecules by setting up simplified models of chain molecules. We use models that have enough of the features of real polymers to prevent their mathematical solution by purely analytical methods. but not so many that they apply to only a few special systems. We expect that the properties found will be broadly characteristic. and we are particularly interested in the limit of very long chains.

The major tool of our investigation is computer modeling. and we use three classes of algorithms: Monte Carlo methods for the equilibrium properties of polymer ensembles. Monte Carlo methods for the solution of certain stochastic dynamical models, and molecular dynamics for the investigation of strictly atomic models. These models are complementary. In particular, the first provides an economical algorithm for providing sets of initial conditions for the second two. As always, we are interested in technical questions as well as getting numerical results. Currently, we are investigating the properties of a methnd called reptation in which chain molecules move at random in a snakelike tashion.

Applications. Better theoretical understanding of conformational and dynamic effects in polymers is needed in their use as structural materials and in studies of biological macromolecules.

M. H. Kalos et al.

COMPUTATIONAL MODELING -- QUANTUM MANY-BODY SYSTEMS

Our work is directed at exploration of computational methods in quantum many-body problems. The scientific problems are drawn from low temperature physics (properties of helium fluids and crystals). from nuclear 
physics (properties of neutron and nuclear matter), and from chemistry (properties of large molecules). We have succeeded in producing methods and results which are computationally and physically more nearly exact than ever before.

The calculations are formulated explicitly as many-body simulations and are in turn of two types. In variational calculations. trial functions which approximate either ground state wave functions or density matrices are formulated and used in variational principles. The resulting many dimensional integrations are carried out by Monte Cario methods. Exact methods use integral equation formulations equivalent to the Schrodinger or Bloch equations: these integral equations are solved by different Monte Carlo methods.

Applications. This research is important for fundamental understanding of quantum fluids and crystals including effects of superfluidity. There are physical and chemical systems that exhibit quantum mechanical effects on a bulk level. For example. the behavior of liquid helium at low temperatures is a direct consequence of quantum effects. Superconductivity of electricity is another example that directly relates to energy technology. Quantum many-body investigations impact significantly upon chemistry.

M. H. Kalos et al.

COMPUTATIONAL MODELING -- CHEMISTRY I

Quantum Chemistry.

Recently. a new program was initiated to simulate a many-fermion system by a Monte Carlo variational method. This research will aid the development of compact and accurate correlation functions for many electron. atomic. and molecular systems. The principal advantage of the method is that the interelectronic distance, rij. may be included directly in the wavefunction without adding significant computational complexity. In addition. other quantities of physical and theoretical interest such as electron correlation functions and representations of coulomb and Fermi "holes" are very easily obtained.

M. H. Kalos

\section{COMPUTATIONAL MODELING -- CHEMISTRY ॥}

Chemical Kinetics

This research deals with the computer simulation of the individual collisions of atoms and molecules that result in chemical reactions. One approach to this problem that will be used is to numerically solve the classical equations of motion for the reaction of interest. Initially. the types of reactions studied will be collisions between atoms and molecules in the gas phase. We will be particularly interested in reactions that are believed to occur in combustion systems.

The considerable interest in the elementary reactions that occur in combusting systems stems from the desire to understand and be able to model these systems on the molecular level. The rate constants of the 
component reactions are often not accessible to experimental measurement, so a computer calculation can be of great assistance in giving estimates of unknown rate constants. Other types of information available from a trajectory calculation are differential cross sections of the scattered product, vibrational-rotational distributions of the products, the activation energy to reaction. and whether vibrational enhancement of the reaction rate occurs. In reactions, where collision complexes form, the properties of the complex can be studied.

Applications. The detailed understanding of macroscopic chemical reactions. for example. those in combustion systems, requires elucidation of the reaction mechanisms at the microscopic level. Through these calculations predictions have been made both on systems accessible to experiment and on important intermediate step reactions that as yet cannot be observed.

P. A. Whitlock

COMPUTATIONAL METHODS OF NEUTRON TRNSPORT

We are using and refining Monte Carlo methods to study problems in reactor physics. The initial emphasis will be on the improvement of correlated sampling methods for the calculations of perturbations. We will undertake study of flux ratios and Doppler effect in water reactors.

M. H. Kalos

22. ADVANCED COMPUTER CONCEPTS

$\$ 250,000$

03 Jacob T. Schwartz. Max Goldstein. Ralph Grishman. Ed Franceschini

A. Gottlieb

ADVANCED LANGUAGE CONCEPTS

For some years we have been developing a high-power programming tool (the SETL language) specialized for the rapid prototyping of complex software systems. This language is now operational both on the VAX cunder DEC/VMS and Unix) and on the CDC Cyber system. Our experience with it indicates that it improves programming efficiency by a factor of 4. A tutorial describing our new programming techniques, and also a comprehensive collection of advanced combinatorial. scheduling. symbolic manipulation. and geometric algorithms is being prepared. SETL has been used to implement a very advanced code optimizer. a compiler for the full Ada language (work supported by DOO), and various other software packages including a FORTRAN compiler. graphics language compiler. assembler for a highly parallel computer, on-line editor. etc.

Because of its very high-level. mathematical flavor. EETL appeare to be an ideal language for studies of program verification, and recently our work has begun to shift into this area. This has triggered attempts. still preliminary. to build a powerful set-theoretically oriented proof verifier. We have also undertaken a series of theoretical studies in this area. which have led to many new decision algorithms for sublanguages of set theory. As our ideas on verifier structure 
develop. we expect a powerful proof and program-verification structure to emerge.

\section{J. T. Schwartz}

\section{DISTRIBUTED SYSTEMS}

Distributed systems will play a significant role in the use of computers for scientific research in the Department of Energy. Our work focuses on the implications of a geographically dispersed. multicomputer environment on communities of scientific users. In addition. the role of the small computer in large-scale computational modeling is being studied.

The approach being taken is essentially experimental and consists of:
a. providing a variety of computer facilities to the CMCL commun- ity of users.
b. acquiring and devising means to easily enable communication among the various hardware and software elements comprising the collection of facilities, and
c. discovering and implementing access methods that reduce the impact on the user of the complexity inherent in multi- computer environments.

Analysis and planning has started for an advanced interconnection method for local network use, suitable for dissimilar hosts. Similar activities in research laboratories and in industry are being tracked. Unfortunately, no totally satisfactory solution to this problem has emerged.

The ARPANET access system is being rèvised to increase the communications bandwidth between the front-end and the VAX. The new design will permit the User Telnet and User FTP functions to be moved to the VAX.

\section{E. Franceschini}

\section{VERY HIGH PERFORMANCE PARALLEL COMPUTERS}

The rapid development of VLSI technology is making it feasible to construct highly paraliel computing assemblages involving thousands or tens of thousands of tightly linked computing elements, all cooperating for the rapid solution of single tasks. Machines of this class could attain instruction rates of tens or hundreds of billions of instructions per second. To make such machines effective, appropriate hardware and software design choices are required. A key hardware issue is the design of an interconnection network capable of moving data at very large rates between processors and memory. This network must also support very high speed interprocessor synchronization problems. Key software Issues are the definition of data structures and operating systems which allow the concurrent operation of very large numbers of cooperating processors. and of programming language features which make it possible for existing code libraries to be adapted for massively parallel execution.

We are currently trying to develop a detailed engineering design for an interconnection network supporting the advantageous software studies have been based. Numerous scientific applications codes are 
being studied for parallelizability. and we expect our underștanding of parallelization techniques and of testing and debugging methods for highly parallel programs to improve steadily. We are beginning to design and implement very highly parallel operating systems. As our hardware design explorations progress, we expect to come into position to build a 'micro-ultracomputer'. probably a 32-processor microchip assemblage.

J. T. Schwartz

23. SPECIAL PROJECTS/

$\$ 100.000$

04 RESEARCH COMPUTING FACILITY Max Goldstein. Ed Franceschiri!

The Research Computing Facllity (RCF) provides the computational resources needed to support the ongoing basic research in Applied Mathematical Sciences. It serves as a testbed for the evaluation and deployment of new computing capabilities as well as serving as the hub of the Electronic Mail System. Maintenance and development of the RCF. including integration and evaluation of emerging computing technologies. is a focal point for collaborative interaction with other DOE research sltes and with researchers in computer science throughout the university and industrial communities. 
OAK RIDGE NATIONAL LABORATORY

Mathematics and Statistics Research Department

Computer Sciences Division

P. O. Box $Y$

Oak Ridge. Tennessee 37830

24. ANALYTICAL AND NUMERICAL METHODS

$\$ 350,000$

D. A. Gardiner. R. C. Ward.

A. D. Solomon. S.-J. Chang

Applied Analysis

Research in applied analysis is concentrated in two areas. moving boundary problems and materials science. and is performed by applied mathematicians in consultation with other scientists and engineers in the subject matter fields.

The work on moving boundary problems is focussed on the qualitative and quantitative analysis of phase change problems, principally arising in heat and mass transfer. Analytical and numerical tools for analyzing and solving these problems will be developed. The emphasis will be on processes with complex boundary conditions. strong nonlinearities, and coupled phenomena. Our techniques used to derive analytical approximations to physical properties of the processes will be studied with respect to these more complicated problems.

Research in the area of materials science is directed toward the development and application of mathematical methods to model and to analyze the physical properties of materials in their solid states. The investigation will be centered on the effect of the distribution of dislocations to the fracture of metals with respect to the newly discovered dislocation-free zone at the crack tip. Our dislocation- free zone model of fracture provides basic understanding on metal fracture in terms of the dislocation emission mechanism at the crack tip. The development of the augmented space method for analyzing amorphous solids and disordered alloys will continue.

Computational Mathematics

The focus of this work is on problems involving sparse matrices in the areas of systems of linear equations. eigenvalue problems, and least squares approximations. The techniques that we have developed for solving sparse least squares problems will be examined for their applicability to solving sparse square systems of linear equations. Variations of the Bunch-Kaufman algorithm and the Lanczos formulation of the conjugate gradients algorithm will also be investigated. Our work on the Lanczos algorithm with respect to linear. quadratic and higher order sparse eigenvalue problems will continue. Software will be produced that is sultable for incorporation into dynamic analysis software packages for analyzing energy producing facilities. Our work on sparse least squares problems applicable to certain earthquake prediction studies, energy distribution problems. energy prospecting. etc.. will be extended to include underdetermined and rank deficient problems and solution updating schemes. Numerical linear algebra problems in computational statistics. such as the determination of independent and dependent parameters in multivariate analysis problems, and in compartmental analysis, such as the stable computation of steady state solutions to closed. Leontief economic models, will àlso be investlgateo. 
25. INFORMATION. ANALYSIS TECHNIQUES

D. A. Gardiner, T. J. Mitchell.

V. E. Kane. K. O. Bowman isc

\section{Statistical Methods}

Research in statistical methods is directed toward the solution of newly discovered or unsolved problems in the analysis of large data sets and in the evaluation of large scale computational models. The work is performed by statisticians in collaboration with numerical analysts and computer scientists as well as with other scientists at local installations, universities and other DOE laboratories. Major statistical topics involved are statistical estimation. experimental design, and multivariate analysis.

The aim of the work in statistical estimation is to develop theory and derive algorithms for computing the uncertainties in model outputs and to contrast these with asymptotic results. Research in experimental design includes the derivation of and experimentation with. screening procedures for Identifyirig important parameters in physical and economic models. The work in multivariate analysis involves the investigation of methods and derivation of algorithms for solving multivarlate problems of classification. variable selection and transformation such as arise in the analysis of large data sets.

Also included in this task are efforts; in collaboration with other statisticians in the Applied Mathematical Sciences program to write the planning document. "Program Directions for Research in Statistical Methods." 
BATTELLE PACIFIC NORTHWEST LABORATORIES

Statistics Section. Energy Systems Department

Computers \& Info. Systems. Engineering Physics. Dept.

P. O. Box 999

Richland. Washington 99352

26. INFORMATION ANALYSIS TECHNIQUES $\$ 500,000$

02

R. A. Burnett, J. J. Thomas. M. D. Erickson.

R. J. Littlefield

\section{Statistical Methods}

The objective of this task is the development of new statistical methods and an improved capability for Analysis of Large Data Sets. Integrated statistics and computer science research is being focused on improved methodologies in statistical analysis and visual display techniques. This work includes the investigation of density estimation. graphical partitioning. discriminant analysis. and other statistical methods as applied to large complex data sets. The data analysis process for large data sets is itself, being analyzed to determine the relative impact of data set size on the choice and sequence of analysis steps. The Research Computing Facility (RCF) at PNL provides a statistical laboratory environment for the development of new data manipulation. analysis. and display methods and the demonstration of those methods on large energy-related data sets. A review panel of expert statisticians and computer scientists from DOE laboratories. universities and government agencles meets annually to guide and evaluate the research and to help disseminate progress to the scientific community.

\section{Data Management}

Effective statistical analysis of large data sets requires efficient specialized data management techniques. This effort involves the development and evaluation of data management tools especially tallored to the requirements of interactive analysis of large data sets. These tools center upon an integrated set of functionally independent data manipulation modules to create. access. and modify data and data descriptions stored in a common self-describing file format. This work includes an evaluation of the suitability of various data dictionary/directory systems for large statistical data bases. Such a system must be able to catalog user-defined data structures and map these structures onto lower-level data vectors stored in selfdescribing files. Tools to interactively search. sample. transform. sort. subset. and edit large data sets will continue to evolve. with particular emphasis on the realization of advanced concepts such as virtual subsets and parallel data flow operations.

\section{Display and Analysis Systems}

The extraction of meaningful information from large volumes of energy related data is of vital and increasing importance to DOE. Today's advanced computer systems can be a tremendous aid in obtaining this information. but only to the extent that powerful computational tools are made usable and readily accessible to the data analyst. Current humancomputer communication techniques inhibit the data analysis process hy forcing the analyst to concern himself with computer-related details. Another common limitation is the lack of capabllity to visualize complex data 
relationships and interact with these graphical representations.

To meet these needs, a research effort in Display and Analysis Systems is ongoing at PNL. A major goal is the development of effective humanmachine communication techniques in the form of adaptable user interfaces and interactive data displays and the application of these techniques to the analysis of large data sets. Semiautomated methods for the synthesis of complex interdisciplinary data analysis tools are also being investigated. These techniques are boing developed and demonstrated on a VAX 11/780 minicomputer system and a high resolution color raster display device. which form the basis of the AMS Research Computing Facility (RCF) at PNL. 
PRINCETON UNIVERSITY

Department of Statistics

Princeton. New Jersey

18. INFORMATION ANALYSIS TECHNIQUES Peter Bloomfield. John Tukey

Interactive Graphical Data Analysis

We are developing improved techniques for interactively analyzing numerical data. A major task is the refinement of current data analysis software and the development of new algorithms. The emphasis is on the effective use of graphical outputs to communicate results to the data analyst. This will require exploration of graphical techniques for presenting the results of otherwise conventional analyses. and also the development of new analysis techniques for which the results are essentially graphical.

We will also define a computer configuration that takes advantage of new mid-sized machines to provide powerful support for interactive data analysis. This will be acquired during the second year and will be our primary equipment for software development.

In the first year progress was made on new types of robust estimates of location called "compromise" estimates. Two types of estimates were studied: a special kind of $M$-estimate called a psi-compromised $M$-estimate. and a gap-compromised estimate which has as its underlying foundation the same working mechanism which has made many $M$-estimates so successful. namely. the downweighting of observations that are extreme with respect to the rest of the data. The estimates discussed here are constructed to do well when the data are from one or two distributions.

The procedures developed can be generalized easily for optimization over any finite set of distributions. An obvious compromise of this sort would be one among the distributions Gaussian, slash, and one-wild that compose Tukey's three corners. 
PURDUE UNIVERSITY

Mathematical Sciences

West Lafayette. IN 47907

27. ANALYTICAL AND NUMERICAL METHODS

$\$ 40,800$

01 John R. Rice

Numerical Solution of Elliptic Partial Differential Equations

ELLPACK is a cooperative project led by John Rice at Purdue University for the study and development of software and methods for solving elliptic problems. Cooperating institutions include the University of Texas. Yale. Harvard. the National Bureau of Standards. Northwestern, and Bell Labs. The project includes the ELLPACK system which has about 30 software modules for all or some of the steps in sulving an elliptic problem. One can naturally state an elliptic PDE and request that it be solved by une (ur mure) of the modules. The objectives of this system are (a) to support extensive performance evaluations of numerical methods. (b) to solve elliptic problems of moderate size. (c) to develop prototypes of large scale software systems for elliptic problems, and (d) education. In the $1981 \mathrm{FY}$ a new version of the ELLPACK system will become operational which will be standardized. thoroughly documented, and portable. New software modules that will be added include general plecewise polynomial collocation. higher order difference methods. blending function methods and multigrid methods. Systematic performance evaluations will be made for (a) methods for 3D problems on rectangular domains. (b) the effect of derivatives in the boundary conditions on various methods' performance. (c) methods for 20 problems with general domains. Research will continue on the development and analysis of new methods that may eventually be included in the ELLPACK system. 
WILLIAM MARSH RICE UNIVERSITY

Department of Mathematical Sciences

Houston. Texas 77001

28. ANALYTICAL AND NUMERICAL METHODS

$\$ 45.000$

Richard A. Tapia. John E. Dennis

Extension of Quasi-Newton Methods to Constrained Optimization and to General Systems of Nonlinear Equations and Inequalities

This research is a continuation of work to extend quasi-Newton methods for unconstrained to constrained optimization problems and to construct effective quasi-Newton methods for large scale problems. Considerable time will be spent on global strategies for constrained optimization. The work summarized here is not based on altering existing quasi-Newton methods to make them applicable to important problems in numerical optimization. Rather, it follows the more ambitious approach of identifying the principles behind the successful methods and then applying those principles to tailor new algorithms for specific classes of problems. Although the long term research goals of the investigators are primarily concerned with the nonlinear programming problem. much of this work is concerned with methods which are new even in the unconstrained case. An objective is to develop computationally effective methods and a primary target is to reduce the cost of the linear algebra involved in each iteration even at the expense of some increase in the number of iterations that would be necessary to solve the problem by more traditional quasi-Newton methods. Thus this class of methods could be useful only in those cases when forming certain coefficient matrices and solving the resulting linear systems is a significant part of the cost of an iteration. Even in such cases enough algebraic work must be saved at each step to make up for any increase in the number of iterations and in associated calls to user supplied function subroutines. The work is progressing. 
SCIENCE APPLICATIONS. INC.

80 Mission Drive

Pleasanton. California 94566

30. ANALYTICAL AND NUMERICAL METHODS

$\$ 65.000$

01

R. J. Gelinas

\section{Computational Analysis of Combustion Processes with} Moving Finite Elements

A four year Moving Finite Element (MFE) research program will direct principal focus upon research and development of the MFE method in 2 dimensions and also continue a smaller level of research on selected topics in 1 dimension which are supportive to our 2-D research. A hallmark of this research effort is its strong collaborative structure that applies the joint investigative efforts of Professor Keith Miller and graduate students in the Mathematics Department at the University of California, Berkeley. and of the research group in basic and applied mathematlcs at sclence Applicatione. Inc.

The first year's efforts are as follows:

1. Undertake the development of an initial experimental computer program (entirely research oriented at this stage) for testing the MFE method in 2-D. A highly modular and flexible structure will be used. based upon the selected basis functions and nodal grids.

2. investigate node control methods. This will include analysis of numerical and nodal behavior for specific classes of PDE's which would include advection dominated and diffusion dominated equations. particularly with respect to PDE's in conservation form.

3. Undertake the solution of linearized equations for the nonlinear ODE's of the MFE in 2-D. A band matrix LU decomposition package may be tested initially.

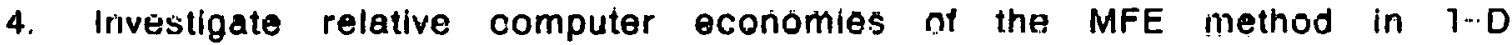
relatlve to existing alternative $P D E$ methods for selected test problems and solutions of comparable numerical quality for the respective PDE methods.

Research in subsequent years will follow and expand on the logical leads observed in the first year's research. 
SANDIA NATIONAL LABORATORIES. ALBUQUERQUE Applied Mathematics Research Department P. O. Box 5800

Albuquerque. New Mexico 87185

31. ANALYTICAL AND NUMERICAL METHODS $\$ 165,000$

01

L. F. Shampine. D. E. Amos. R. J. Hanson

Mathematical Software

Research is being done in the areas of computational mathematics and software engineering. A package. DEPAC. of the best codes for solving ordinary differential equations (ODEs) is being written. Great effort has gone into its software design. In addition. major algorithmic advances are being made. The three best methods for non-stiff ODEs are Adams. Runge-Kutta, and extrapolation. Adams has the big advantage of an interpolation capability. It has been discovered how to provide the capability in the Runge-Kutta code of DEPAC. A similar idea greatly improves the situation for extrapolation. An extrapolation code is being written for DEPAC. The biggest software issue in the solution of ODEs has been recognizing stiffness. Great strides are being made with this task. Besides the obvious convenience to users. problems are solved more efficiently. Work continues on a portable LP code for some large scale problems. A linear least squares code with equality constraints and bounds on the varlables is also being developed for large scale problems. Finally, research and development is underway on a package for Bessel functions of complex argument and non-negative orders. A continuing effort on exponential integrals will provide needed results on Bessel functions and repeated integrals of $K o(x)$. In the area of software engineering. work is underway to complete and document the Text Exchange System being developed to make exchange of source codes easier on different machines. 
SANDIA NATIONAL LABORATORIES, LIVERMORE Computation Department

P. O. Box 969

Livermore. California 94550

32. ANALYTICAL AND NUMERICAL METHODS

$\$ 325.000$

01

Robert J. Kee. S. Margolis.

L. Petzold. M. Smooke

Our research spans three topical areas: Applied Analysis" (bifurcation phenomena in physical systems). Computational Mathematics (stiff ordinary differential equations (ODE) and boundary value problems) and Numerical Methods for Partial Differential equations (PDE) (error control and accelerated convergence). An important aspect of our efforts is the development and application of the mathematical techniques needed to solve the stiff equations which model combustion phenomena. In the Applied Analysis area we dre studying the bifurcation of solutions to premixed flame problems. Current work centers unl extension of high activation energy asymptotic analysis to include several chemical reactions. Applications of this work include predictions of flame stability limits and oscillating flame betiavior

Our ODE research deals primarily with the numerical solution of stiff systems of differential algebraic equations (DAE). We are currently deriving the algorithms needed to solve general systems of DAE's and we have begun to write a general purpose DAE computer code. Since many PDE's are solved via a method of lines approach, our DAE work is coupled closely with our work on the numerical solution of PDE's. In solving systems of PDE's we are especially concerned with error control and computational efficiency. To achieve error control we are developing the methodology to adaptively place mesh points in regions of the solution having large gradients or curvature. To achieve efficiency we seek sparse matrix iteration strategies which converge rapidly yet have a large domain of convergence for stiff problems. 
STANFORD UNIVERSITY

Systems Optimization Laboratory

Department of Operations Research

Stanford, CA 94305

33. ANALYTICAL AND NUMERICAL METHODS

$\$ 368,000$

George B. Dantzig. Richard W. Cottle

Walter Murray

\section{Systems Optimization Project}

Tho Eystems Opllmization Project is one of the principal activities of the Systems Optimization Laboratory (SOU. Department of Operations Research. Stanford University. The work of the project is directed toward the development of effective numerical methods for practical optimization and equilibrium problems arising in the energy field and other areas. Behind this overall objective. research at SOL includes a range of different aspects: definition and characterization of useful problem categories: creation and design of algorithms: thorough study of the mathematical and numerical properties of algorithms: implementation with sound numerical and programming techniques: and evaluation and comparison of algorithms. Currently research, is proceeding in the following areas: methods for staircase linear programs: analysis of conjugate gradient and sparse quasi-Newton techniques for unconstrained optimization: extensions to the MINOS/AUGMENTED code for large scale constrained optimization: development of several projected Lagrangian methods for nonlinearly constrained optimization problems: development of truncated Newton methods: extension of quadratic programming subproblem methods to solve large problems: and methods for sparse linear least-squares. 
STANFORD UNIVERSITY

Computer Science Department

Stanford. California 94305

34. ANALYTICAL AND NUMERICAL METHODS

$\$ 91.000$

01

Gene H. Golub

\section{Numerical Analysis}

The purpose of this task is to devise numerical procedures for solving a variety of numerical problems which arise in solving mathematical problems associated with the energy needs of the nation. A byproduct of many of these investigations is mathematical software. An area of special concern is the fitting of data in several situations. An especially useful technique for snlving such problems is orthogonalization methods which are frequently more stable than the usual procedures and allows for perturhations in the data. In this category the following problems are being consdidered: 1) total least squares estimation. 2) least squares tensor product spline fitting. and 3) extensions of the variable projection algorithm for solving nonlinear least squares problems. The variable projection algorithm is very useful for solving problems which involve sums of exponentials. Work is progressing to extend this algorithm for analyzing multivariate data and problems with special structure. It appears that this technique will be very useful for situations where the data is contaminated by errors in the fixed variables as well as the observed variables. A procedure is being developed for solving the biharmonic equation on a rectangular region. The basic approach is to break the problem up into the sum of two problems. one of which is easy to solve. The splitting of the operator in this fashion allows for an extremely fast procedure and it appears that this method will be of some importance in solving the Navier-Stokes equation for large Reynolds numbers. A robust plece of software is expected from this project. 
STANFORD UNIVERSITY

Computer Science Department

Stanford. California 94305

35. INFORMATION ANALYSIS TECHNIQUES

Werner Stuetzle. Jerome $H$. Friedman

Gene H. Golub

\section{Projection Pursuit Algorithms for Graphical Data Analysis}

The aim of this research is to develop a new class of nonparametric methods for multivariate regression. classification and density estimation. The common property of the proposed algorithms for those three problems is that they attempt to build a model for the multivariate structure of the data based on the information contained in suitably chosen lower cusually 2 -) dimensional projections. Projections of the data are cheap to compute and easy to interpret, and the central role of projections in the algorithms makes them ideally suited for graphical output. Two lines or research are currently being pursued. On the one side. the algorithms are implemented in noninteractive Fortran programs with printer-plotter graphics: in this case projections revealing the structure of the data are sought by numerical optimization. The other focus of the research is implementation of the algorithms on a computer graphics system that allows the user to interactively search for structured projections of the data. The interactive graphics allows one to discover features of the data (clustering. concentration on low dimensional manifolds. outliers. ...) that would easily go undetected otherwise. 
TEXAS TECH UNIVERSITY

Institute for Numerical Transport Theory

Department of Mathematics

Lubbock. Texas 79409

36. ANALYTICAL AND NUMERICAL METHODS

Paul Nelson. H. Dean Victory. Jr.

\section{Seventh International Conference on Transport Theory}

The Seventh International Conference on Transport Theory was held at Texas Tech University, in Lubbock. Texas, during March 17-20. 1981. It was attended by approximately forty mathematicians. nuclear engineers and physiclsts. most of whom are actively pursuing research in transport theory. Six of the attendees were from forelyn cuurilites. including two from Poland. and one each from Germany. India. Italy. and Yugoslavia. in addition a paper from the People's Republic of China was presented in absentia. A total of 30 papers were presented. The proceedings are to appear as a special issue of the journal Progress in Nuclear Energy. with a presently scheduled publication date in the spring of 1982. 
UNIVERSITY OF CALIFORNIA, BERKELEY Center for Pure and Applied Mathematics Berkeley. Callfornia 94720

\section{ANALYTICAL AND NUMERICAL METHODS} R. J. Fateman. W. Kahan

Research in the Design and Implementation of a Comprehensive Facility for Scientific Computation

The goal of this work is to incorporate the extensive experience of the investigators into a unique scientlfic software facility representing the uncompromisingly best capabilities which can be made available in the new breed of large address space. low cost. high performance computers (typifled in current product lines by the Digital Equipment Corporation VAX $11 / 780$ and the Prime 400). The first principal objective is to ogranize a body of numerical procedures in such a way that they may be invoked automatically by processes which accept symbolic and algebraic specifications from a user, and produce combined symbolic and numerical output. The second principal objective is to make the algebraic system as flexible and useful as possible in this context. and to integrate it successfully into a man machine design which provides operating system. language. and algorithm support. 
UNIVERSITY OF CALIFORNIA. LOS ANGELES

School of Engineering and Applied Science

Computer Sclence Department

Los Angeles, Callfornia 90024

\section{ADVANCED COMPUTER CONCEPTS \\ Gerald Estrin. Bertram Bussell \\ Danlel Berry. David Martin}

$\$ 250.000$

03

Methodology for the Synthesis of Information Processing Systems

This research has a goal of introducing better computer supported methods to help designer realize information systems that can be accurately predicted to satisfy prestated customer requirements. Given DOE's continuing need for much higher pertormance systems to solve erieryy related problems. the importance of being able to predict performance before writing programs and before building machines is self evident.

The UCLA methodology has been designed to support both a "top down" partitioning procedure and a "bottom up" composition procedure. In both cases. however, the procedures are requirement-driven so that attention can be continually focused on reducing the gap between a designer's declared intent and the actual behavior of a system. For many historical reasons the UCLA system has incorporated tools in its methodology to deal with concurrent processes. This aspect increased in importance with the dramatic advances in VLSI technology. The synthesis tools are needed for design of the VLSI devices themselves. to manage design of systems. containing very large numbers of such devices and to design interacting programs running on such devices.

The SARA system has acted as the focal point for our research investigations into improved methods for design of complex information systems. The system can be characterized as an evolving set of related tools created in the form of interactive computer programs and data structures and reachable by designers from remote terminals. These tools are intended tó be consistent with support of a philosophy and methodology exposed in publications by our group. However. the design support tools can be used independently. Most of the tools that have been developed are useful in models representing hardware or software at a relatively high level of abstraction. We are still striving to bridge such models to code or to sumlcunductór cells. 
UNIVERSITY OF COLORADO

Computer Science Department

Boulder, Colorado 80309

39. ADVANCED COMPUTING CONCEPTS

$\$ 99.900$

Lloyd D. Fosdick, Leon J. Osterwell

\section{Software Engineering - TOOLPACK}

This research is aimed at improving the environment for the production and maintenance of mathematical software. We are seeking to achieve this through the use of a well coordinated set of software tools which may be accessed by the user with a simple command language. The tools included in this system are a lexer. parser. a program formatter. a syntax driven editor, a static analyzer. a dynamic analyzer. and a macro preprocessor. along. with other lower level tools. These tools are designed to operate on FORTRAN source programs and may be modified easily to accommodate a limited set of FORTRAN extensions. This system is intended to be portable. Our work is being carried out in collaboration with workers at several other places: Argonne National Laboratory. University of California at Santa Barbara. Purdue University. Jet Propulsion Laboratory. Bell Laboratories. International Mathematical and Statistical Libraries (IMSU. Inc.. and the Numerical Algorithms Group (NAG) Ltd. 
DOE Applied Mathematical Sciences

FY 1981

UNIVERSITY OF DELAWARE

Department of Mathematics

Newark. Delaware 19711

$\$ 34.500$

01

40. ANALYTICAL AND NUMERICAL METHODS

Robert Gilbert. Allan Jeffries

Mathematics of Oil Production and Transportation

The subject under study is the mathematics of modeling thermal secondary oil recovery techniques. either by in situ combustion or hot water or steam injection. The study also includes inverse methods for determining the location and shape of interfacial strata between water. oil. and gas. The investigators are exploring modifications of a simplified in situ combustion model. taking into account both the oil phase and the gas phase and including the effects of gravity segregation of condensate.

Also. a systematic study of the derivation of equations that characterize the macroscopic motion of fluids in a porous elastic medium will be undertaken.

$-52-$ 
UNIVERSITY OF FLORIDA

Database Systems Research and Development Center

Department of Computer and Information Sciences

Gainesville. Florida 32611

41. INFORMATION ANALYSIS TECHNIQUES Stanley Y. W. Su. Sham Navathe. Don Batory

$\$ 49.500$

02

Logical and Physical Modeling and Design of Scientific and Statistical Databases for Energy Research

This research focuses on the logical and physical modeling and design of scientific and statistical databases (SSDs) and has two specific areas of emphasis. First. it investigates characteristics of a powerful data model to a) provide for the modeling of a variety of data types. aggregation functions and statistical summary data. b) allow for an expliclt definition of the semantics of scientific and statistical data to simplify the user's task in formulating queries. and $c)$ provide powerful data and query mapping facilitles to bridge multiple users' models cof exlsting and proposed SSOs in a distributed environment) with a global conceptual model. Second. implementation techniques are studied to a) identify efficient ways of processing SSDs in terms of retrievals. updates. etc.. and b) facilitate the handling of compressed data, variable length data and various data types which are characteristic of SSDs. A systematic approach to logical and physical design of SSDs will be investigated. The research is being carried out by members of the Databasee Systems Research and Development Center. University of Florida. 
UNIVERSITY OF ILLINOIS

Computer Science Department

Urbana. Illinois 61801

42. ANALYTICAL AND NUMERICAL METHODS $\$ 220.000$

C. W. Gear. D. S. Watanabe

Computational Mathematics - Ordinary Differential Equations

The project is developing theory, algorithms and software for the solution of a variety of problems in differential equations and their applications. Among the difficulties being investigated are problems with highly oscillatory solutions. problems with discontinuities, and problems whose solution components have very different rates of variation. Experimental. software has been developed for the oscillatory problem, and appears to be sufficiently successful that we have begun implementation of a pre-production package for distribution. Recent experiments on a new method for discontinuity location encourage us to begin preparing a gerieral package. Applications of numerical methods to the partial differential equations arising in semiconductor modelling are being studied. Currently we are investigating the static modelling of 2-dimensional unipolar and bipolar semiconductor devices. A key component is the solution of large. sparse. quasi-linear equations. Work is nearly complete on a package for simulating MOSFETs and JFETs. Additional work is underway on the development of tools for the preparation of mathematical software. 
UNIVERSITY OF ILLINOIS

Department of Computer Science

1304 West Springfield Avenue

Urbana. Illinois 61801

43. ADVANCED COMPUTING CONCEPTS

$\$ 125.000$

David Kuck. Duncan Lawrie

Design Evaluation and Use of High Performance Computer Systems

Despite the vastly improving technology available to the computer industry. there are still application areas which are beyond the capabilities of conventional computers. To get sufficient performance for these applications requires the design and construction of very high performance computers. "supercomputers". with organizations. including parallel. pipeline. and multiprocessing computers. However, programs must undergo significant changes in their structures before they. can take advantage of the high performance offered by these computers.

Over the past ten years we have developed a "supercompiler". Parafrase. which analyzes fortran programs and is capable of restructuring them to execute more efficiently on very high speed computers. The present grant funds two activities. First, the development of a computer architecture simulation facility which. together with Parafrase. will enable us to evaluate the performance of proposed new computer organizations before they are actually built. This facility includes two simulators which are currently under development. One is designed to simulate array (SIMD) computers. The other deals with multiprocessor (MIMD) organizations. This latter simulator is driven by address traces generated from programs which have been transformed by Parafrase to take advantage of multiprocessing. Both of these simulators will eventually be combined into an easy to use facillity which will allow users to get performance estimates for their computers and applications programs. The second activity is to use Parafrase to analyze a number of current application programs to determine the best way to restructure them for current computers. By examining a few of these programs. we have found that they employ constructs that cause them to miss getting significant performance gains from high speed computer architectures. By using Parafrase we can identify those constructs and suggest alternatives that allow full utilization of these computers. 
UNIVERSITY OF TEXAS

Department of Computer Science :

Austin. Texas 78712

44. ADVANCED COMPUTER CONCEPTS

\author{
James Browne. G. J. Liporski. M. Malek
}

High Performance Parallel Computing

This research program combines theoretical/modeling and empirically oriented approaches to varistructured and multiphased parallel formulation of computations. The thrust is not only to find and describe procedures for parallel computing but to evaluate the mechanisms and methodologies proposed by driving significant problems all the way to execution on the highly flexible parallel hardware system - the Texas Reconfigurable Array Computer (TRAC).

The prototype configuration of TRAC consists of 16 processors and 81 memories-1/O units. scheduled to be operational in the Fall of 1981. TRAC provides varistructure and resource partitioning. which support the concept of designing an architecture for an algorithm rather than mapping an algorithm to an architecture. The TRAC interconnection network implements processor-memory and processor-processor circuits and also a high performance packet switching capability. This allows study of process execution models ranging from pure shared memory intercommunication to completely disjoint and distributed address spaces.

There are three principal research areas: software for varistructured reconfigurable multiprocessor architectures. multi-phase parallel formulation of significant complete problems. and further development of varlstructure reconfigurable network based computer architectures. 
UNIVERSITY OF TEXAS

Texas Institute for Computational Mechanics

Austin. Texas 78712

45. ANALYTICAL AND NUMERICAL METHODS

$\$ 65.000$ Graham F. Carey. David M. Young

Iterative Solution and Finite Element Analysis in Computational Mechanics

This research concerns the use of iterative solution algorithms in conjunction with finite element methods for computing numerical solutions of transient and nonlinear problems in mechanics and heat transfer. Particular focus is directed towards the solution of very large scale. complex problems by means of advanced computer architectures exploiting vectorization and parallelism.

In the theoretical and numerical investigations we examine accuracy and error estimates for certain classes of transport problems - for example. in heat transfer, reservoir simulation, and Navier-Stokes theory. Related interesting questions concerning special techniques. such and "lumping" and "upwinding" are being examined. Moreover, we seek to determine the influence of such strategies on the structure and behavior of the approximate problem and solution iterative techniqués.

The analysis of transient problems is based on a semidiscrete formulation in which selection of appropriate system integrators play an important part in determining the overall efficiency of the computation. In the investigation of iterative techniques we study the properties of the methods for the classes of problems indicated. acceleration of the methods and tailoring specific methods to exploit special features of emerging computer architectures of current interest. Numerical case studies will help provide an evaluation of the technical innovations arising from the study of finite element and iteration methods. 
UNIVERSITY OF WISCONSIN

Computer Science Department and

Statistics Department

Madison. Wisconsin 53706

\section{INFORMATION ANALYSIS TECHNIQUES $\$ 152.800 \quad 02$ \\ David J. DeWitt. Haran Boral \\ Computer Sciences Department \\ Doug Bates. Statistics Department}

\section{Database Machines for Large Statistical Databases}

This project deals with the Irivestigation of database machinco for supporting efficient access to large statistical databases. Most. If not all. database machine designers have concerilrated on the efficient support of operations on relational databases. Statistical operations on relational databases (e.g. aggregates) have received little attention. No body of research exists on the execution of any type of operations on statistical databases. The goal of this project is the implementation of a prototype statistical database machine.

To accomplish this goal an "algorithmic" approach is being utilized. This process begins by accurately characterizing the operations used in querying statistical databases. Next.' the best uniprocessor algorithms for these operations will be collected and analyzed. The result of this analysis will be used as a baseline against which the expected performance improvement of parallel algorithms (which we will design) will be measured. Our parallel algorithms will be analyzed using a generalized multiprocessor model so that the analysis is independent of any architectural constraints. The results of this analysis. will point to the strengths and weak points of each algorithm and will help identify those primitive operations that must be provided by the hardware.

In addition to the evaluation of the performance of various algorithms we will investigate the suitability of various file organizations and compression schemes for the implementation of the underlying storage structure. Finally, various mass storage technologies will be examined and characterized with respect to factors such as cost. suitability to parallel access. access time. etc. At this point we shall have a sufficient understanding of query execution and storage devices to enable us to begin to design an architecture whilch perrills hlyli peiformance access to vary large statistical databases. 


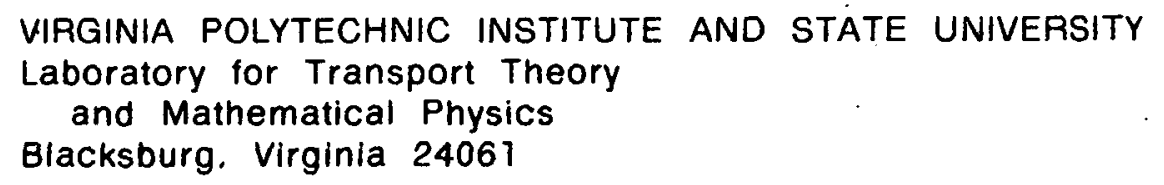

\section{ANALYTICAL AND NUMERICAL METHODS \$0(in FY 81) 01}

William Greenberg. P. F. Zweifel

\section{Applied Mathematics of Transport Theory}

A number of projects have been initiated since this research program began in the last quarter of 1980. These include a study of the possible collisional restabilization of a marginally linearly unstable plasma. The analysis is carried out using a modified. linearized Fokker-Planck modification of the linearized Vlasov equation to describe collisions. Another project involves the analytical study of multi-component gas flows. which has involved both a derivation of the relevant equations (using a BGK collsion model) and their solution via resolvent integration techniques. A third project has generalized existence theorems for nonlinear evolution equations: application of these results to the Boltzmann equation provided a generalization of previously derived existence theorems. A further project involves Monte Carlo studies of lattice spin systems. A program has been written and is now operational, and will be used to study phase transitions in systems with infinitely many ground states, but a finite number of "dominant" ground states. Finally. a new project involves a study of generalized transport equations where the transport operator is replaced by an arbitrary self-adjoint operator. Existence. uniqueness and full and partial range completeness are being studied for a variety of such operators. 
YALE UNIVERSITY

Computer Science Department

New Haven. CT 06520

48. ANALYTICAL AND NUMERICAL METHODS $\$ 32.000 \quad 01$

Tony F. C. Chan

Numerical Methods for Nonlinear Elliptic Eigenvalue Problems

Nonlinear eigenvalue problems are problems of the form $G(u, p)=0$, where $u$ belongs to some Banach space and $p$ is a scalar parameter. Many important problems in the physical sciences can be cast in this form. Among these are problems in elasticity. structural mechanics, fluid dynamics, chemical engineering. meteorology. and plasma physics. These problems often possess interesting nonlinear bifurcation phenomena, for example, the existence of limit points and bifurcation points.

The ultimate objective of this project is to produce efficient and reliable software for such problems. We are investigating both the numerical and software aspects that arise. The baslc method we are using is the arclength continuation method of H. B. Keller. Among some of the important computational issues are: fast location of singular points. automatic step size algorithms, improvement of the basic Newton process and fast linear algebra, solution methods for large elliptic problems. A software package is being developed. 
Amos. D. E.

Bates. D.

Batory. D.

Berry, D.

Betancourt, 0 .

Bloomfield, P.

Boral, $H$.

Bowman. K. $\mathrm{O}$.

Boyle, J. $M$.

Browne. J.

Bucher. 1.

Burnett, R. A.

Burridge, $R$.

Bussell. B.

Butler, D.

Buzbee. B.

Carey. G. F.

Carison, B.

Carr, D. B.

Chan. T. F. C.

Chang. S. J.

Char, B. W.

Chorin, A.

Cody. W. J.

Coleman. T. F.

Concus, $P$.

Cook, J. M.

Corones, J.

Cottle. R. W.

Cowell, W. R.

Dantzig, G. B.

Davison, $M$.

Defacio. B.

DeWitt, D. J.

Dendy. J. E.

Dennis, J. B.

Denni3. J. E.

DiPerna. $R$.

Dongarra, J.J.

Dritz, K. W.

Erickson. M. D.

Estrin, G.

Fasel. J.

Fatemain, R. J.

Feldman, M. (B.

Foley. J. D.

Fosdick, L. D.

Franceschini.

Frederickson. $P$.

Friedman, J. $H$.

Fritsch. $F$.

Gabriel, J. R.
43 Garbow. B. S.

10

58 Gardiner. D. A.

53 Gautensen, A. K.

35. 36

50

26. 27

Gear. C. W.

9

54

39

58

36

10

\section{6}

\section{8}

37

26

50

18

18

57

.9

37

60

35

10

20

10

10

20

10

9
45

45

10

46

Gelinas, R. J.

42

Gilbert. $R$.

Goldstein. C.

Goldstein. M.

Golub. G. H.

Gottlieb. A:

Greenberg. W.

Greiman. W.

Grishman. R.

Grunbaum. F. C.

Hall. D. E:

.Hall. D. L.

Hanson. R. J.

Hillstrom, K. E.

Hindmarsh. A.

Hiromoto, $\mathrm{R}$.

Ho. J.

Holmes. H.

Hyman. J. M.

Isaacson. $E$.

Jeffries. A.

Jordan. T.

Kahan. W.

Kalos, M.

Kane. V. E.

Kao. C. S.

Kaper. H. G.

Kee. R. J.

Keller. H. B.

Kreuger, $R$.

Kuck. D.

24 Lawrie. D.

26. 29 Leaf. G. K.

10.11

Liporski. $G$.

Littlefield. R. J.

Lusk. E. L.

Luskin. M.

Lyness. J. N.

Malek. M.

Margolis. S.

15 Martin. D.

51 Matkowsky, B. J.

32. 33,34

McBryan. 0 .

McGraw, J.

47 McMaken. $\mathrm{H}$.

22 Messina. P.

10 Michael, G.
52

12

32. 34

46. 47

32

59

20

32

20

21

37

43

10

22

18

12

20

18

26. 29

52

18

49

26, $30,31,32,33$

36

12

10

44

14

9

55

55

26

10

56

37

10

26. 29

10

56

44

50

10. 25

26. 27

22

. 9

10. 11

22 
Minkoff. M. 10

Mitchell, T. J. 36

More', J. J. 10

Murray. W. 45

Navathe, S. $\quad 53$

Nelson, $P$. $\quad 48$

Nichols, B. 18

Nicholson. W. L. 37

O'Dell, M. 21

Osterweil. L. J. 51

Overton. M. 26. 28

Pasciak, J. 12

Petzold, L. $\quad 44$

Dunng. C. 20

Rice. J. R. 40

Hobbins. $H . \quad 12$

Rodrigue. G. 22

Roediger. G. 11

Royston, R. 10, 11

Saffman, P. G. 14

Schwartz, J. T. 32, 33, 34

Shampine. L. F. 43

Shoshani, A. 20

Smith. B. T. 10

Smooke. M. 44

Solomon. A. D. 35

Soreneen, D C. In

Stewart. $H . \quad 12$

Strauss. $H$. 26. 27

Stuetzle. W. 47

Su. S. Y. W. 53

Sventek. J. 21

Tapia, R. A. 41

Thomas. J. J. 37

Tukey. J. $\quad 39$

Van Ryzin, J. $\quad 12$

Varga, R. S. $\quad 76$

Veroff, R. L. 10

Victory. H. D. 48

Wang. P. S. $\quad 16$

Ward. R. C. 35

Watanabe. D. S. 54

Watson, K. 17

Whitlock. P. 26. 32

Widlund. O. 26. 28

Winker, S. K. 10

Wos. L. T. 10

Young, D. M. 57

Zweifel, P. F. $\quad 59$ 
United States

Department of Energy

Washington, DC 20545

Official Business

Penalty for Private Use, $\$ 300$
Postage and Fees Paid

U.S. Department of Energy

DOE-350

\section{U.s.mall}

